\title{
"IN QUESTO", IL TEATRO GLI SCENARI DELLA COMMEDIA DELL'ARTE
}

\author{
Roberto CUPPONE ${ }^{1}$
}

- RIASSUNTO: Il segreto della commedia dell'arte è stato nel paradosso di Maschera e Improvvisazione, Tipo Fisso e Testo Variabile, Conservazione e Innovazione. Per questo è stato nei secoli esaltata o denigrata: considerata reazionaria dalla Rivoluzione francese e esaltata come rivoluzionaria dai Romantici. Nel Novecento, Copeau, Mejerchol'd, Mnouchkine e Fo hanno dovuto fare i conti con questa ambivalenza. Dunque ciò che oggi si chiama commedia dell'arte è "reazionario" o "rivoluzionario"? "Popolare"o "populista"?

- PAROLE-CHIAVE: Commedia dell'arte; Maschera; Improvvisazione.

\section{Introduzione ${ }^{2}$}

\footnotetext{
1 DUTARS - Diploma Universitario in Tecniche dell'Arte e dello Spettacolo - Venezia - 30124 Italia.

2 Il presente saggio sviluppa i contenuti dell' introduzione a una dispensa per gli studenti per il corso di Storia dei Generi Teatrali Folclorici e Popolari, DUTARS, Facoltà di Lettere e Filosofia, Università di Ca' Foscari, a. a. 2000-2001, primo semestre; la dispensa contiene inoltre i seguenti scenari e passi di rilevanza teorica cui d'ora in poi si farà qui riferimento.

Troiano 1568

Massimo Troiano, ["Ditemi quanti personaggi furono..."], in Discorsi delli triomfi, giostre, apparati e delle cose più notabili, fatte nelle sontuose nozze dell'Illustrissimo et Eccellentissimo signor duca Guglielmo, primo genito del generosissimo Alberto Quinto, conte Palatino del Reno e duca della Baviera alta e bassa, nell'anno 1568, a' 22 di febraro, di Massimo Troiano da Napoli, musico dell'Illustrissimo et Ecc. signor duca di Baviera. In Monaco, appresso Adamo Montano MDLXVIII;
} 
I circa mille "scenari” ("soggetti”, “canovacci”) di commedia dell'arte fino ad oggi conosciuti costituiscono un corpus di documenti scarsamente studiato nella sua globalità e molto eterogeneo. Appunto in questa eterogeneità stanno la sua ambiguità e insieme la sua ricchezza: un ventaglio di exempla di scrittura teatrale che attraversa i secoli, le culture attoriche e soprattutto si articola ogni volta diversamente con la scena: in modo riassuntivo, progettuale, allusivo, tra de-scrizione e pre-scrizione, elaborando gerghi espliciti e impliciti, sedimentando e istituendo di fatto una "scienza" della costruzione dello spettacolo che è alla base della scrittura teatrale degli ultimi quattro secoli.

Dil brano in oggetto si trova oggi in Studi sul teatro veneto fra Rinascimento ed età barocca, a cura di Maria Teresa Muraro, Firenze: Olschki, 1971; e in Flaminio Scala, Il teatro delle favole rappresentative, a cura di Ferruccio Marotti, Milano, Il Polifilo 1976, 2v.

Li quattro finti spiritati 1611

Flaminio Scala, Li quattro finti spiritati, giornata XXXIII, in Il teatro delle favole rappresentative, overo la ricreatione comica, boscareccia e tragica, divisa in cinquanta giornate, Venezia: Pulciani 1611; l'edizione completa moderna è a cura di Ferruccio Marotti (F. Scala, Il teatro ..., cit.).

Prologo del Finto marito 1618

Flaminio Scala, Prologo, in Il finto marito, Venezia, Baba 1618; i due prologhi della commedia si trovano oggi in Carmine Jannaco, Stesura e tendenza letteraria della commedia improvvisa in due prologhi di Flaminio Scala, in "Studi Secenteschi”, I, 1960, p.195-207; in F. Scala, Il teatro ..., cit. con introduzione del curatore Marotti; in Roberto Tessari, Commedia dell'arte: la Maschera e l'Ombra, Milano, Mursia, 1981; l'intera commedia si trova in Laura Falavolti, Commedie dei comici dell'Arte, Torino, Utet, 1982.

L'ateista fulminato c. 1650

L'ateista fulminato, quarto scenario della raccolta Ciro Monarca. Dell'opere regie, Roma, Biblioteca Casanatense, ms. 4186; pubblicato in Francesco De Simone Brower, Ancora una raccolta di scenari, in "Rendiconti della reale Accademia dei Lincei", 1901, v.10, s.V, p.391-407; di nuovo, dalla trascrizione di De Simone Brower, in Enzo Petraccone, La Commedia dell'Arte. Storia, tecnica, scenari, Napoli, Ricciardi, 1927, p.374-82; in Anton Giulio Bragaglia, Commedia dell'Arte, canovacci inediti raccolti e presentati da A. G. Bragaglia, Torino, Il Dramma, 1943, p.25-34; in Giovanni Macchia, Vita avventure e morte di Don Giovanni, Torino, Einaudi, 1978.

Quattro finti spiritati XVII.

Quattro finti spiritati, terzo scenario in Scenari per il teatro San Cassiano, Venezia, Biblioteca Correr, codice 1040; oggi in Gli scenari Correr. La commedia dell'arte a Venezia, a cura di Carmelo Alberti, Roma, Bulzoni, 1996; oltre che in alcune tesi di laurea, che Alberti segnala (p.12)

Trapolaria 1699 - 1734.

Placido Adriani, Trapolaria, diciassettesimo scenario in Selva ovvero zibaldone di concetti comici, raccolti da D. Placido Adriani, ms., Perugia, Biblioteca Comunale, D 16; copia dell'omonimo scenario di Perrucci da Giovanni Della Porta, in Andrea Perrucci, Dell'arte rappresentativa premeditata e all'improvviso, Napoli, Mutio, 1699 (edizione moderna a cura di A. G. Bragaglia, Firenze, Sansoni, 1961).

Les vingt deux infortunes d'Arlequin 1738 - post 1762.

[Carlo Goldoni], Les vingt deux infortunes d'Arlequin, Paris, Bibliothèque Nationale, Fond Français, v.9254 dei mss., ff. 1-23; oggi in Carlo Goldoni, Tutte le opere di Carlo Goldoni, a cura di G. Ortolani, v.XII (traduzione mia). 


\section{Un corpus immaginario}

La estensione effettiva di questo corpus è variamente definibile, ${ }^{3}$ a seconda che si comprendano o no gli scenari francesi, della Comédie Italienne o delle foires, quelli goldoniani (e, una volta superato tale spartiacque, perché non gli scenari romantici?), ${ }^{4}$ che si considerino o no i racconti, gli estratti, gli "argomenti", ecc. Se poi si aggiungono la grande variabilità di stili, di autori (professionisti e dilettanti; attori e accademici), di finalità delle raccolte manoscritte e pubblicate; che senso, storico o critico, può avere la comparazione di questi "mille" scenari? Lo stesso che parlare di una unica "commedia dell'arte": nessun senso, grande interesse. La consistenza di questo corpus infatti è la stessa che viene attribuita al fenomeno storico cui si allude con l'espressione "commedia dell'arte": un monstrum, definito e idealizzato a posteriori, fino a diventare petizione di principio, schietta utopia, proiezione sul passato di ombre (luci) del teatro attuale. ${ }^{5}$

D'altro canto queste scritture hanno innegabilmente molto in comune: esattamente tutto quello che non sono. Non sono (non vogliono essere) un testo disteso, che si rapporti alla scena come trans-scrizione completa dell'elemento verbale (sottintendendo che questa sia possibile, mentre ovviamente non lo è, anche nel caso del cosiddetto teatro di tes-

\footnotetext{
3 Allardyce Nicoll è il primo ad elencare 760 titoli (in Masks, mimes and miracles: studies in popular theatre, New York: Harcourt Brace, 1931); seguito da Kathleen Marguerite Lea che ne riprende 700 in Italian popular comedy: a study in the Commedia dell'Arte, 1560-1620, with special reference to the English stage, Oxford: Clarendon Press, 1934; Vito Pandolfi ne pubblica un elenco leggermente più esteso, di cui, con poche eccezioni, dà anche i riassunti e un breve commento panoramico (in $L a$ commedia dell'arte. Storia e testi, Firenze: Sansoni, 1958, v.V, cap.XIV, Gli scenari dell'arte); Thomas F. Heck, in quella che a tutt'oggi è la più estesa bibliografia ragionata della commedia dell'arte (con tutte le contraddizioni e le lacune che derivano dalla definizione stessa dell'oggetto), a partire da Nicoll, elenca 820 titoli, con rispettiva provenienza, in Commedia dell'arte. A Guide to the Primary and Secondary Literature, New York, London: Garland Publishing, 1988 (Appendix. Scenari of the commedia dell'arte: a title index). Non si può non citare infine l'impresa incompiuta di Zorzi, che aveva annunciato la trascrizione di tutte le principali raccolte italiane esistenti per farne una analisi comparata, sia storica che testuale (Ludovico Zorzi, La raccolta degli scenari italiani della commedia dell'arte, in Alle origini del teatro moderno. La commedia dell'arte, atti del convegno di studi, Pontedera, 28-29-30 maggio 1976, Bulzoni, Roma 1980, p.104-15).

4 Cfr. Roberto Cuppone, L'"invenzione" della commedia dell'arte, Torino: C.I.R.V.I. (Centre Interuniversitaire de Recherche sur le Voyage en Italie), 1998, 2 v. (Id., Le Théâtre de Nohant; e George Sand, Maurice Sand, Théâtre inédit, documents et dessins); e Id., CDA. Il mito della commedia dell'arte nell'Ottocento francese, Roma: Bulzoni, 2000.

5 Per la revisione metodologica in atto nel campo degli studi sulla commedia dell'arte, partita vent'anni fa dalle geniali intuizioni di Taviani (F. Taviani, M. Schino. Il segreto della commedia dell'arte. Firenze: La casa Usher, 1982), v. oggi R. Cuppone, CDA..., cit..
} 
to). Non sono (non vogliono essere) operazioni legittimate da un Autore, invece che dalla sedimentazione delle esperienze e dagli "effetti" che sono in grado di suscitare. Non sono (non vogliono essere) opere d'arte autonome e compiute, piuttosto che strumenti di lavoro in comune e in progress. ${ }^{6}$

Ma se ciò è vero, se gli scenari sono più interessanti per ciò che non sono piuttosto che per ciò che sono (e in effetti le poche "regole" di composizione che li ispirano hanno già condannato alla sterilità più di un tentativo di interpretazione strutturalistica); più per la parte sommersa che per quella emersa, come degli iceberg; allora il loro studio è questione di primaria importanza, non solo storica ma anche critica, non solo pertinente un (in)determinato periodo storico, ma tutta la storia del teatro. Proseguendo nella metafora, il loro studio si può definire come il problema di dove collocare la linea di galleggiamento della Scrittura (o di emersione del testo) nel mare della Rappresentazione.

Dunque, nell'attesa asintotica di delimitare l'arco storico di riferimento e di stabilire il livello formale per cui uno scenario può essere definito tale, l'insieme di tutte queste scritture, questo corpus alla deriva, resta tenuto insieme da criteri più analogici che analitici; e il loro numero, mille, è immaginario tanto quanto la loro continuità effettiva.

\section{Le mille e una favole rappresentative}

Ciò che effettivamente conta, di questo corpus, non è tanto il peso quanto i movimenti interni, sia in senso sincronico che diacronico: come una singola storia si fa rappresentazione, e nello stesso tempo come viene riletta e trasformata a distanza di chilometri e/o di secoli. Ciò che interessa è questa alternanza di ripetizione e differenza, per usare una espressione di Deleuze, ${ }^{7}$ che riproduce in scala macroscopica il processo creativo che avviene per ogni singola maschera a livello intermedio, e per ogni singolo lazzo a livello microscopico.

6 Vedi a questo proposito Ferdinando Taviani. La composizione del dramma nella Commedia dell'Arte. In: Quaderni di Teatro, IV, n.15, p.151-71.

7 Gilles Deleuze, Différence et répétition, Paris: José Corti, 1970 (Bologna: Il Mulino, 1971); con introduzione di Michel Foucault. "Se la ripetizione è possibile, essa inerisce al miracolo, piuttosto che alla legge": è un'affermazione che potrebbe valere tanto per un esperimento di fisica quantistica che per una improvvisazione teatrale. 
Questi movimenti pongono radicalmente in discussione il concetto di scrittura (e di creazione) teatrale come gesto individuale: non a caso i romantici (Charles Nodier, Théophile Gautier, George e Maurice Sand), che inventarono la commedia dell'arte come genere, credendo di vederla reincarnata nei teatrini del boulevard, collaborarono con essi scrivendo canovacci di cui negarono la paternità e anzi teorizzarono questa militanza anonima in nome dell "auteur collectif" del teatro popolare. E, in fondo, alcune questioni critiche ormai leggendarie, come la questione omerica o la meno fortunata questione scecspiriana, basano i loro argomenti su questa imbarazzante, eterna (dis)continuità tra le fonti e l'opera.

Mille e una "favole rappresentative", dunque, come un grande non libro effettivamente scritto, una gigantesca e plurisecolare (verrebbe da dire: antropologica) scenoteca in cui attingere scene primarie, secondarie, ennesime in un telescopico movimento di un teatro alla ricerca di sé, come l'adulto cerca sé nella propria infanzia e lo storico la società nella sua preistoria.

E non è un caso che l'etimo stesso di rappresentazione contenga il prefisso iterativo, ed anzi sia parola pullulante di prefissi, che nella loro successione sembrano descrivere esattamente il processo della messa in scena, comune alla psicanalisi e al teatro: ogni rappresentazione essendo una $r i-a d$ - prae - sent - atio, un riaffiorare ( $r i)$ motivato $(a d)$ nel presente (prae) di esistenze (esse) ancora attive (atio).

\section{Un DNA della rappresentazione}

Troiano 1568 può essere scelto come il primo di questi exempla. ${ }^{8} \mathrm{Si}$ tratta del resoconto di una "commedia all'improvviso a l'italiana" rappresentata alla corte di Baviera in occasione delle nozze del duca Guglielmo, da una compagnia sorta all'occasione riunendo alcuni "professionisti”, perlopiù musicisti (i due animatori essendo appunto Massimo Troiano e Orlando di Lasso) e alcuni cortigiani; senza donne, dato che la parte di Camilla venne cortesemente sostenuta dal marchese di Malaspina en travesti (quattro anni dopo la prima comparsa di una donna, Lucretia senese, in un contratto di compagnia).

8 Già lo fa, ad esempio, Marotti in F. Scala, Il teatro..., op. cit. 
La natura d'occasione dello scenario si legge anche in una certa sproporzione dei tre atti, in alcuni sottointrecci non ben risolti, nella elementarità di alcuni lazzi ("il Zanne pigliò in spalla lo suo patrone e, voltizzanno a guisa di rota di molino, quanto più ebbe cielo di durare girò, e similmente il Pantalone al Zanne fece lo medesimo"), nella netta prevalenza dell'elemento musicale, che apre e chiude ogni atto. Ma in rapporto alla gestazione di una nuova forma di scrittura per il teatro, questo resoconto è esemplare perché è esposto in tono narrativo, intercalato dalle classiche domande di un platonico interlocutore, ma nello stesso tempo troppo dettagliato e compiuto per confondersi con il resto del resoconto. La misura di questa ambiguità fra cronaca e critica, fra racconto e Racconto, è data in pieno dall'alternanza talvolta incongrua di passato remoto e presente indicativo.

Già nel momento stesso di farsi racconto, una rappresentazione di maschere diventa dunque quasi ineluttabilmente schema: nel momento che il narratore, già protagonista dello spettacolo, si prova a sintetizzarlo, si trova inevitabilmente a riproporlo nella sua forma di paradigma, di exemplum, un po' esperienza e un po' petizione di principio. Analogamente a quanto succede anche sul versante iconografico, dove la riproduzione di momenti di commedia dell'arte istituisce letteralmente una forma d'arte autonoma, più imparentata all'odierno racconto per fumetti che all'arte figurativa in genere. ${ }^{9} \mathrm{D}$ 'altro canto, non dobbiamo dimenticare che la stereotipizzazione (vero e proprio modo di produzione del nascente teatro professionale) pervade tutti i livelli della creazione: dal lazzo, alla maschera, al soggetto, con assonanze e forze di attrazione reciproche che non sono mai casuali. Così già Troiano, che probabilmente non presume ancora di dare alle stampe una commedia, non può esimersi dal citare proprio come punti di forza i suoi topoi ("pensando a i maccarù $e$ sbruffadèi, che per lo adietro mangiar fatto l'avea"), e d'altro canto, come si conviene all'epica popolare, di inframmezzare il racconto con la testimonianza diretta dei suoi effetti ("giuro che, a quante comedie stato io sono, mai vidi tanto di core ridere").

Dunque, la questione se e quando lo scenario sia una scrittura "preventiva" o "consuntiva" dello spettacolo riguarda in fondo gli storici, che

\footnotetext{
9 Si veda ad esempio la nota sequenza del Recueil Fossard, da alcuni riferita a un coevo spettacolo dei Gelosi (Recueil de plusieurs fragments des premières comédies italiennes qui ont été représentées en France sous le règne de Henry III. Recueil dit de Fossard, conservé au Musée national de Stockholm, présenté par Agne Beijer. Suivi de Compositions de rhétorique de Don Arlequin, présentées par P. L. Duchartre, Paris: Duchartre et Van Buggenhoudt, [1928]); o le "caricature" di Callot di poco posteriori (Jacques Callot, Balli di Sfessania, Nancy 1621-1622).
} 
sperano di trovarci indicazioni per rievocare questo eterno fantasma che è la performance; ma da un punto di vista funzionale, non fa molta differenza. Lo scenario si inscrive in un continuum fra la scena e la pagina, in cui la scena travasa, a momenti dati, sulla pagina, spunti, considerazioni, idee, ricordi derivati dalla precedente esperienza del palcoscenico; i termini del "problema" non essendo il Testo e lo Spettacolo, ma la Scrittura e la Rappresentazione; non due astrazioni, ma due "gesti" concreti.

Dunque, come per l'iconografia, anche per questi "scenari" è depistante concentrarsi sul loro valore di verità (sia che la si intenda come documentazione di un'esperienza pregressa, sia che prevalga l'affermazione di un dover essere teorico); che indubbiamente vi si può in qualche misura trovare. Si tratta di forme di espressione autonome, che in questo loro farsi vanno dunque analizzate. Ancora una volta ci aiuta una forma di pensiero metaforico: immaginare lo scenario come una specie di DNA della rappresentazione, memoria di ogni precedente messinscena di quella fabula, ma anche (e proprio in quanto tale) progetto di un nuovo individuo.

\section{Una drammaturgia di azioni}

In questo senso, l'osservazione secondo cui lo scenario incarnerebbe un sistema di produzione teatrale innovativo in sé, ma conservativo in quanto impermeabile alle esperienze degli altri paesi europei contemporanei, potrebbe dunque essere rovesciata: si tratta di un sistema conservativo del sapere teatrale, e in quanto tale innovativo rispetto agli esperimenti degli umanisti. ${ }^{10} \mathrm{E}$ infatti più di uno storico parla di "sperimentalismo" riferendosi al Teatro delle favole rappresentative di Flaminio Scala, prima raccolta a stampa di scenari dell'arte. ${ }^{11}$ Che è anzi due volte "prima", come raccolta e come stampa, essendo preceduta, per quanto ci è dato conoscere, solo da pochi prototipi che sono appunto Troiano 1568, l'analogo resoconto di una recita della Pazzia di Isabella dei Gelosi in occasione delle nozze fiorentine di Caterina di Lorena; e una commedia di inaspettato autore, Galileo Galilei, che ci è pervenuta sia in forma di scenario che di commedia estesa. ${ }^{12}$

10 "Classico" anziché "classicistico"; cfr. F. Taviani, La composizione del dramma .... cit., p.162 e ss.

11 V. l'Introduzione di Ferruccio Marotti in F. Scala, Il teatro... cit.

12 I testi di questi precedenti sono pubblicato in F. Scala, Il teatro... cit. 
A parte i motivi di interesse generale della pubblicazione, bilancio artistico di un attore a fine carriera e documento storico di una generazione che perseguiva una cosciente strategia di autocelebrazione, questa raccolta presenta carattere di novità soprattutto nell'affermazione cosciente di un genere di drammaturgia fondata sulle "azioni". Dove per azione non si intende riduttivamente "gesto", ma unità narrativa convenzionale, che associa e interseca una connotazione temporale (l'azione è innanzitutto qualcosa che accade, evento), un significato causale (ogni azione si definisce tale in quanto capace di suscitare reazione) e finanche una immagine di sfondo, per il suo carattere topico. La piena consapevolezza teorica di questa drammaturgia è espressa in Prologo del Finto Marito 1618, uno dei due prologhi con cui Scala fa precedere la versione estesa di una commedia già presente nella sua raccolta (Il marito, giornata IX): "perché in effetto alle azioni son più simili l'azioni che le narrazioni, e le commedie nell'azioni consistono propriamente et in sustanzia, e nelle narrazioni per accidente".

Dunque il valore dell'azione sta nel fatto che da un lato fonda il meccanismo della creazione teatrale (spiegando meccanicamente lo svolgersi della tanto mitizzata improvvisazione, che altro non era se non una sequenza ragionata di azioni e reazioni); dall'altro è anche garanzia della possibilità che essa venga compresa dal pubblico, poiché l'imitazione della realtà non avviene né solo a livello di testo, né solo di immagine, ma di entrambi in quanto azioni, cioè eventi, agenti, icone nel senso sopra definito.

Questa "drammaturgia di azioni", questo meccanismo collaudato dai nuovi professionisti del teatro, si scopre ben presto tanto fondante e autonomo, che può allontanarsi indefinitamente dalla fabula di partenza, fino al punto di farne a meno: diventa puro intreccio, sequenza senza fine di "colpi di scena", virtuosismo compositivo, come accade in certe pochades ottocentesche. Esemplare in questo senso è Li quattro finti spiritati 1611, non certo lo scenario più conosciuto della raccolta di Scala, ma autentico esempio limite di come l'intreccio, giocando sulla pura convenzione, può portare a esiti paradossali. Diversamente dal topos della Pazzia, in cui fu celebre Isabella Andreini e che alcuni ritengono l'antenato dello strip tease, qui i personaggi diventano "spiritati", mettendo alla dura prova la credulità nonché di Pantalone, del pubblico stesso. Ma se è vero che "i buoni concetti si conoscano dalli effetti, e non da' precetti", 13 l'accettabilità dell'espediente non si giudica a priori, ma dalla sua capa-

13 Prologo del Finto Marito, 1618. 
cità di sortire effetto sul pubblico. E infatti lo scenario procede come un teorema: una volta accettato in convenzione lo "spiritamento" di un personaggio, questo può riprodursi in modo esponenziale, sempre più veloce e immotivato, sugli altri personaggi fino al parossismo narrativo.

\section{"Fisiologia" della maschera}

Vale ricordare che la drammaturgia di azioni è una conseguenza diretta del teatro delle maschere. Un teatro fatto con la maschera non potrebbe altrimenti essere de-scritto o pre-scritto, in quanto la maschera per definizione non pensa, ma agisce; ed anzi essa stessa risponde a quei tre requisiti che contribuiscono a definire l'azione: è evento in quanto contraddizione incarnata fra l'attore e il tipo, fra il vivo e il morto; è agente, in quanto non può non suscitare un effetto; è icona, in quanto rimanda a un sistema di rappresentazione figurato, sia quello della demonologia da cui proviene, sia quello posteriore dell'arte che l'ha interpretata.

Senza dire che anche concretamente, nella sua fisicità, la maschera condiziona palesemente i modi della narrazione; e non solo grazie alla tipizzazione del personaggio, di cui la coazione a ripetere è solo l'aspetto più immediato e superficiale; ma proprio per i limiti oggettivi che pone alla recitazione dell'attore (visivi, acustici, motori), per la tendenza alla segmentazione del movimento del corpo e in genere dell'espressione. La maschera si fa massimamente espressiva nei suoi momenti di immobilità totemica, i cosiddetti "colpi di maschera" con cui l'attore fa precedere la rottura comica - tanto che spesso si cita la marionetta come logico sviluppo di questa segmentazione espressiva (ma erroneamente: non a caso Craig che teorizzò questo sviluppo, ${ }^{14}$ confondendo il significante col significato, la metafora con l'oggetto, non riuscì a produrne un esempio concreto in teatro).

Inoltre la maschera porta al suo massimo grado di iconicità (teatralità) la "apparizione" del personaggio, cioè la sua entrata in scena; e conseguentemente aumenta la significatività della sua uscita. E' forse inutile documentare questa affermazione associandola a livello antropologico, con le "incarnazioni" sciamaniche; a livello storico, con il valore rituale e spettacolare del deus ex machina; o, a livello psicologico, con

14 V. Gordon Craig, Towards a new Theatre. London: Dent \& Sons, 1913. 
l'interpretazione psicanalitica del gioco infantile del rocchetto (del nascondino). Di fatto, nel teatro delle maschere è tale l'importanza emotiva delle entrate e delle uscite, che si fa subito narrativa. Esse determinano il ritmo generale del racconto, ancora prima della (e talvolta nonostante la) velocità dell'eloquio o di altre forme di scansione ritmica dello spettacolo, tanto che la suddivisione per scene dello scenario corrisponde quasi sempre esattamente con la successione delle entrate e delle uscite.

E' in questi modi che, tra fisica e metafisica della Rappresentazione, la maschera si fa portatrice di un tertium convenzionale, di una "fisiologia", intesa come comportamento tendenziale e sperimentato, che si fa subito costituente dei meccanismi di composizione dello scenario.

\section{Teatro di Creazione versus Teatro di Repertorio}

E' dall'incrocio di questi due codici inespressi, una drammaturgia di azioni e una fisiologia della maschera, che si giustifica e si spiega il caratteristico genere di scrittura sintetica degli scenari, la cui condensazione apparentemente paradossale porta a volte a far sottostimare lo scenario e addirittura l'intera raccolta di cui fa parte; sulla base di considerazioni più letterarie che funzionali.

Un esempio di questo genere è fornito dal confronto di Quattro finti spiritati XVII della raccolta Correr, con l'omonimo scenario di Scala. A distanza di pochi decenni, agli sconosciuti comici del teatro veneziano di San Cassian è possibile trans-scrivere una vicenda dallo sviluppo sostanzialmente identico a quella di Scala, con qualche lazzo in più ("Capellino spara la pistola, tutti hanno paura, fugiano, e finisce l'atto" primo), con metà parole e soprattutto con tre personaggi in meno, rinunciando al terzo nucleo familiare (quello che in Scala erano il Capitano Spavento e Arlecchino) e al tormentone di Nicoletto. Non per questo semplificando l'intreccio, anzi complicandolo; dato che qui Coviello riunisce in sé sia la parte di padre che di innamorato frustrato, quelle che in Scala erano rispettivamente di Graziano e Capitano Spavento.

I tre movimenti (nel senso che si diceva prima) che emergono dal confronto dei due scenari - verso una maggiore spettacolarizzazione, una ergonomia della distribuzione, una complicazione dell'intreccio testimoniano di una accresciuta consapevolezza compositiva degli attori, pur nella minore padronanza della lingua rispetto a quella dell'intellettuale Scala; e, qualora riscontrati in altri scenari dello stesso manos- 
critto, porterebbero a rivalutare una raccolta, quella appunto del Correr, fino ad oggi attribuita - anche per scarsità oggettiva di informazioni - a una compagnia di scarso interesse professionale.

E infatti il problema delle fonti degli scenari è quasi sempre affrontato, anche se non apertamente, come una ricerca della "non novità" anziché della in-novazione, del "difetto" anziché del pregio. La prospettiva andrebbe invece capovolta: proprio laddove esistono fonti individuate, è nello scostamento da esse che si può valutare appieno la professionalità e la capacità narrativa dello stesore del nuovo scenario. Ancora una volta si ritorna all'assunto di partenza, che il valore di ogni singolo scenario è tanto più comprensibile quanto più inscrivibile nel corpus di scritture di cui fa parte, pur analogicamente. Ogni nuova rappresentazione aggiungendosi a un teatro che si può definire paradossalmente di repertorio e di creazione nello stesso tempo.

\section{Incredibilmente vero o credibilmente falso?}

Non è un caso, in questo senso, che la storia più raccontata non solo dalla "commedia dell'arte", ma dal teatro in genere, e cioè quella di Don Giovanni, sia stata fino ad oggi materia di studi in molti dei quali questo rovesciamento di prospettiva critica è già avvenuto. Nella letteratura su Don Giovanni, infatti, la ricerca dei "precedenti", da versione a versione, valorizza i minimi scostamenti, in quanto nel loro complesso disegnano gli slittamenti secolari del mito, dal nucleo medievale e penitenziale del conte Leonzio, a quello barocco e controriformistico caratterizzato dalla figura dell'Ateista, fino al libertinismo delle versioni settecentesche e operistiche; o alle problematizzazioni romantiche. ${ }^{15}$

L'ateista fulminato c. 1650, della raccolta Casanatense, è la più antica versione a canovaccio conosciuta del mito di Don Giovanni; propone un antieroe innovativo rispetto alla commedia di Tirso e precursore di Molière per molti aspetti (fra tutti per la scena dell'elemosina al Povero), costituendo un vero e proprio anello di congiunzione fra le due commedie estese, tra Spagna e Francia, tra Rinascimento e Barocco. Ma nell'economia del discorso sulla scrittura è soprattutto interessante perché esemplifica il rapporto dialettico che la "commedia dell'arte" ebbe

15 Esemplare in questo senso è il saggio di G. Macchia, Vita, avventure e morte... cit. 
sempre con le cosiddette unità aristoteliche: dallo sviluppo per quadri alternati, città/campagna, corte/covo dei banditi, che segue un andamento epico, quasi da ballata popolare (con giustapposizioni di scene che talvolta evocano il montaggio cinematografico), si ricava come la preferenza implicita per le unità, spesso riscontrata nei canovacci, non è rispetto di un precetto ma, ogni volta, scelta consapevole di efficacia narrativa. Don Giovanni non sarebbe tale se la sua vicenda non si modellasse sulle "occasioni" che gli si presentano, che per definizione devono essere diverse: donne di diverso ceto, condizione, cultura; incontri con nobili e plebei, perché la sua filosofia di vita è interclassista; una condizione fra omologazione (l'origine nobile) ed emarginazione (la vita da bandito).

Ma la scrittura di uno scenario può arrivare anche più lontano. In genere, si può dire che nella stilizzazione di ogni scenario c'è una potenzialità non sempre espressa appieno: quella di far scivolare il racconto nel surreale senza soluzione di continuità, e nello stesso tempo senza pretesti narrativi. Arlecchino può finire nel mondo della luna senza la necessità che ciò venga giustificato come ricordo, sogno, delirio, flashback; ma semplicemente in battuta, o meglio proseguendo una logica di azioni. Il mondo descritto dal teatro classico sta a quello della "commedia dell'arte" come quello del trasporto lineare sta a un fantastico mondo del teletrasporto: nel canovaccio le categorie di spazio e di tempo sono convenzioni, non postulati, e come tali si possono comprimere e attraversare dallo stargate dell'invenzione poetica.

E dunque, in questo caso, partire da una storia già conosciuta non è un fattore di debolezza ma di forza; non solo e non tanto per il richiamo che essa può avere sul pubblico, ma soprattutto perché, giocando su aspettative già esistenti, senza il vincolo di crearne ex novo, libera l'energia creativa più intrinseca e paradossale del racconto scenico, che si esprime nella vertigine degli intrecci come nella inverosimiglianza delle metamorfosi; in perfetta libertà e artificio, essendo l'azione, l'unità narrativa base, non elemento realistico, ma appunto convenzionale.

Senza voler sfruttare le coincidenze del linguaggio oltre il lecito, è divertente notare come i due aggettivi più frequenti nei titoli degli scenari di Flaminio Scala sono di gran lunga "fido" e "finto": ma se "fido" compare in Il pellegrino fido amante (giornata XIV), Li tre fidi amici (XIX), Li duo fidi notari (XX), Il fido amico (XXXIX); "finto" invece lo troviamo in La finta pazza (VIII), Il finto negromante (XXI), Il finto Tofano (XXIV), Flavio finto negromante (XXVIII), Li finti servi (XXX), Li duo finti zingari (XXXI), Li quattro finti spiritati (XXXIII), Il finto cieco (XXXIV); senza parlare della Mancata fede (XXVII) e di Il creduto 
morto (XXII). Grande lezione di poetica per noi oggi, dopo secoli di dibattito sul realismo nell'arte. L'attore sa, per averlo sperimentato letteralmente sulla propria pelle (o su quella della propria maschera), che la verosimiglianza di un racconto non si misura in assoluto, ma in rapporto alle aspettative del fruitore, preesistenti o suggerite dalla narrazione stessa.

\section{Una scrittura per leggere il teatro}

Dove dello scenario esiste anche una versione estesa, precedente o seguente, dello stesso o di diverso autore, esso diventa una vera a propria chiave di lettura per l'attore che si accinge a rappresentare la commedia. Trapolaria 1699-1734, ad esempio, che nel testo studiato è presa dal manuale di Perrucci del 1699, e da questo è ripresa nell'omonimo scenario della Selva di Adriani (1734), in origine deve la sua fabula a una commedia di Giovanni Battista Della Porta. ${ }^{16}$ Del resto Perrucci per definizione concepisce proprio così lo scenario, che "non è altro che una tessitura delle scene sopra un argomento formato, dove in compendio si accenna un'azione che deve dirsi e farsi dal recitare all'improvviso, distinguendosi per atti e per scene... Questi soggetti o son fatti apposta per rappresentarsi all'improvviso o sono presi da commedie distese dagli antichi o moderni, ridotte a stilo di potersi rappresentare all'improvviso". ${ }^{17}$ Inutile sottolineare quell' "argomento formato", cioè, sottinteso, anche da altri; quella "azione che deve dirsi e farsi" a partire da scenari che possono indifferentemente essere "fatti apposta" o "presi da commedie distese dagli antichi o moderni".

In effetti, l'aiuto più importante che lo scenario può dare alla comprensione della commedia estesa non è ovviamente quello di carattere filologico, ma soprattutto teatrale: una specie di verifica alla luce dell'es-

16 Giovanni Battista Della Porta (Napoli, 1535, ivi 1615), scienziato e autore, pubblicò La Trappolaria nel 1596; sicuramente essa venne rappresentata a Ferrara nel 1614. In genere, la produzione di Della Porta ebbe un ruolo strategico nella evoluzione del corpus degli scenari: le sue commedie, innovative rispetto alle sperimentazioni degli umanisti, si ispiravano spesso alla trame plautine e a loro volta vennero spesso riprese negli scenari degli attori (in particolare L'Olimpia, L'Astrologo, La Sorella, La Fantesca, Il Moro, La Tabernaria, con Pulcinella; La Trappolaria, appunto, Li duo fratelli simili e La Notte); rappresentando forse così il più importante anello di congiunzione fra la commedia latina e il nuovo teatro dei professionisti.

17 A. Perrucci, Dell'arte... cit., p.232. 
perienza (una, o anche molte) degli attori, una sintesi funzionale, un drenaggio della storia alla luce degli effetti che essa è stata effettivamente in grado di produrre in teatro, al di là delle possibili attestazioni di stima sulla lingua, sulle citazioni, sulla morale del racconto.

D'altro canto è anche vero il converso; per comprendere il senso degli scenari e della loro scrittura condensata sono necessarie una frequentazione e finanche la pratica della scena, come insegna l'attore-autore oggi più titolato a sostenerlo, Dario Fo: "bisogna buttarsi in una vera e propria ricerca puntando a decifrare i canovacci, che sono numerosissimi, comparandoli e confrontandoli tra loro, soprattutto per quanto riguarda l'interpretazione, per lo più misteriosa, dei lazzi, con le soluzioni comiche del cosiddetto teatro minore: le farse popolari dell'Otto-Novecento, il teatro di varietà, l'avanspettacolo, gli spettacoli dei clown e perfino le comiche del 'muto'. E' in queste forme del comico che si è riversata gran parte del materiale della commedia dell' arte. Per finire la pratica è il mezzo migliore per imparare a leggere ogni testo teatrale". ${ }^{18}$

In questo movimento di andata e ritorno, dalla Scrittura alla Rappresentazione e da questa di nuovo alla Scrittura, stanno tutta l'attualità e l'intrinseco valore pedagogico degli scenari.

\section{Stereotipo e Archetipo}

Perfino Goldoni, cui sicuramente appartiene Les vingt deux infortunes d'Arlequin 1738 - post 1762, esempio di scenario fra i più tardi, pervenutoci nella trascrizione di qualche attore della Comédie Italienne, attinse a questa immaginaria "scenoteca", alla Biblioteca di Babele del teatro, non a caso a inizio e fine di carriera, come del resto per diversi altri suoi scenari. E la letteratura si è variamente sbizzarrita a imparentare questo scenario a La sorella di Placido Adriani, a sua volta ispirata all'omonima commedia di Della Porta, a sua volta di derivazione plautina.

Spesso si è parlato di "decadenza" degli scenari in epoca immediatamente pregoldoniana, nel senso di uno sviluppo del lazzo da mimico a verbale, da organico a eccentrico; di una crescente prevalenza del lazzo sul soggetto, della digressione sulla progressione - ma su questo vi sarebbe molto da dire, essendo proprio nella digressione, che è appunto

18 Dario Fo, Manuale minimo dell'attore. Con un intervento di Franca Rame, Torino: Einaudi, 1987, p. 110 . 
trasgressione del soggetto principale, la vitalità teatrale del canovaccio, il suo hic et nunc. Gli scenari goldoniani, in particolare quelli che sopravvissero alla stesura in commedia e quindi si trasmisero come tali, nei repertori degli attori, dall'Italia alla Francia e di nuovo in Italia, ${ }^{19}$ sembrano essere in controtendenza. Sommessamente, inaspettatamente, il Goldoni francese della Comédie Italienne fa buon viso a cattivo gioco. Chiamato a ritornare in qualche modo sui suoi passi, a recedere dal suo progetto di riforma della commedia per tornare a una scrittura sommaria, prende alla lettera questo ritorno alle origini: sceglie alcuni dei soggetti più topici di ogni epoca, gli "accidenti", le "metamorfosi", i figli delle maschere, la perdita di memoria, tutti già da decenni nel repertorio "europeo" della più importanti compagnie dell' Arte sopravvissute, come quella di Sacchi; ${ }^{20}$ e attraverso di essi, e anche grazie a una discreta dose di autobiografia (particolarmente significativa, in tre di essi, è la figura di un Arlecchino emigrante, che come Ulisse brama il rientro a casa) attinge a un nuovo livello di significazione, quasi archetipica.

E' forse tempo di ripensare Goldoni fuori dagli stereotipi di riformatore e accanito avversatore della "commenda dell'arte": perché di fatto, paradossalmente, fu forse l'unico autore della "commedia dell'arte", senz'altro l'autore più importante fra quanti abbiano scritto degli scenari. E dunque per quanto abbia inteso superarne la forma, lo fece a partire da una profonda intelligenza del loro meccanismo di scrittura, che anzi portò al suo livello più alto di consapevolezza. Dallo stereotipo all'archetipo, appunto.

La commedia di per stessa è rivisitazione di situazioni che si ripetono (si pensi a espressioni come "commedia degli equivoci", o "commedia umana") e dunque galleria di storie stereotipe, mentre è proprio della tragedia, in genere, la rivisitazione delle forme archetipe; nella prima gioca la variabile spazio, orizzontale e reversibile, nella seconda gioca la variabile Tempo, verticale e irreversibile. La grande commedia è quella capace di generare l'illusione che nello Stereotipo si delinei l'Archetipo. E che, nel dubbio, fa ridere.

Il corpus degli scenari della "commedia dell'arte", con Goldoni, compie l'ultimo passo verso il mito.

19 Fra questi, oltre a quello studiato, Il figlio di Arlecchino perduto e ritrovato, Le metamorfosi di Arlecchino, La bague magique; oggi in Carlo Goldoni, Tutte le opere... cit., vol. XII.

20 Per maggiori informazioni su questo repertorio europeo, v. R. Cuppone, I figli delle maschere, ovvero l'eredità impossibile. Sipario, numero speciale "Goldoni e l'arte scenica", set. 1993, p.32-41. 


\section{Appendice}

Prospetto delle principali raccolte conosciute di scenari

\begin{tabular}{|c|c|c|c|}
\hline Data & Titolo vulgato & $N$. & Collocazione del manoscrito elo pubblicazione \\
\hline 1568 & [Massimo Troiano] & 1 & $\begin{array}{l}\text { in Massimo Troiano, Discorsi delli trionfi, giostre, } \\
\text { apparati, e delle cose più notabili fatte nelle sontuose } \\
\text { nozze dell'Illustrissimo et Eccellentissimo Signor Duca } \\
\text { Guglielmo. München: Montano, 1568; poi Dialoghi di } \\
\text { Massimo Troiano..., Venezia } 1569\end{array}$ \\
\hline XVI & $\begin{array}{l}\text { [Modena, Estense, } \\
\text { Scenari modenesi] }\end{array}$ & 2 & $\begin{array}{l}\text { Modena, Biblioteca Estense, ms. I, } 740 \text { (a S.8,14) } \\
\text { (oggi in Emilio Re, Scenari modenesi, in Giornale } \\
\text { Storico della Letteratura Italiana, LV, 1910, p.2 e ss.) }\end{array}$ \\
\hline $\begin{array}{l}\text { inizio } \\
\text { XVI }\end{array}$ & $\begin{array}{l}\text { [Modena, Archivio } \\
\text { di Stato, } \\
\text { Scenari modenesi] }\end{array}$ & 9 & $\begin{array}{l}\text { Modena, Archivio di Stato: documenti teatrali, busta V } \\
\text { (oggi in Emilio Re, Scenari modenesi, in Giornale } \\
\text { Storico della Letteratura Italiana, LV, 1910, p.2 e ss.) }\end{array}$ \\
\hline 1611 & $\begin{array}{l}\text { Flaminio Scala, } \\
\text { Il teatro delle } \\
\text { favole } \\
\text { rappresentative }\end{array}$ & 50 & $\begin{array}{l}\text { Flaminio Scala, Il teatro delle favole rappresentative, } \\
\text { overo la ricreatione comica, boscareccia e tragica, } \\
\text { divisa in cinquanta giornate. Venezia: Pulciani, } 1611 \\
\text { (oggi in Flaminio Scala, Il teatro delle favole } \\
\text { rappresentative. A cura di Ferruccio Marotti, Milano: } \\
\text { Il Polifilo } 1976,2 \text { v.) }\end{array}$ \\
\hline $\begin{array}{l}1618 \\
1622\end{array}$ & $\begin{array}{l}{[\text { Roma, }} \\
\text { Casanatense, } \\
\text { Basilio Locatelli] }\end{array}$ & 103 & $\begin{array}{l}\text { Della scena de soggetti comici di B. L. R. [1618] } \\
\text { \& Della scena de soggetti comici et tragici di B. L. R. } \\
{[1622]} \\
\text { Roma, Biblioteca Casanatense, F. IV, 12-13 (ora } 1211 \\
\text { \& 1212) }\end{array}$ \\
\hline $\begin{array}{l}\text { c. } \\
1650\end{array}$ & $\begin{array}{l}{[\text { Roma, }} \\
\text { Casanatense, Ciro } \\
\text { Monarca] }\end{array}$ & 48 & $\begin{array}{l}\text { Ciro Monarca - Dell'opere regie } \\
\text { Roma, Biblioteca Casanatense, ms. } 4186\end{array}$ \\
\hline XVII & $\begin{array}{l}{[\text { Firenze, }} \\
\text { Magliabecchiana] }\end{array}$ & 22 & $\begin{array}{l}\text { Firenze, Biblioteca Nazionale, Magliabecchiana II, i, } \\
10 \text { e il corrispondente ms. in Biblioteca Riccardiana, } \\
\text { codice } 2800 \\
\text { (oggi in Adolfo Bartoli, Scenari inediti della commedia } \\
\text { dell'arte. Contributo alla storia del teatro popolare italiano. } \\
\text { Firenze: Sansoni, 1880) }\end{array}$ \\
\hline XVII & $\begin{array}{l}\text { [Roma, Vaticana } \\
\text { Barberiniana] }\end{array}$ & $\begin{array}{l}9 \\
19\end{array}$ & $\begin{array}{l}\text { Dal n. } 6 \text { intitolati Selva di nuove comedie } \\
\text { Roma, Biblioteca Vaticana, fondo Barberiniano } 3895\end{array}$ \\
\hline
\end{tabular}




\begin{tabular}{|c|c|c|c|}
\hline XVII & [Venezia, Correr] & 51 & $\begin{array}{l}\text { Scenari per il teatro San Cassiano } \\
\text { Venezia, Biblioteca Correr, codice } 1040 \\
\text { (oggi in Gli scenari Correr. La commedia dell'arte a } \\
\text { Venezia, a cura di Carmelo Alberti, Roma: Bulzoni, } \\
\text { 1996) }\end{array}$ \\
\hline Data & Titolo vulgato & $N$. & Collocazione del manoscrito e/o pubblicazione \\
\hline XVII & [Roma, Corsiniana] & 100 & $\begin{array}{l}\text { Raccolta di scenari più scelti d'istrioni divisi in due } \\
\text { volumi } \\
\text { Roma, Biblioteca Corsiniana, mss. } 45 \text { G.5 (49) e } 45 \\
\text { G.6. (51) }\end{array}$ \\
\hline $\begin{array}{l}\text { c. } \\
1700\end{array}$ & $\begin{array}{l}\text { [Napoli, } \\
\text { Casamarciana] }\end{array}$ & 183 & $\begin{array}{l}\text { I. Gibaldone comico di varj suggetti di comedie ed } \\
\text { opere bellissime... del Conte di Casamarciano, } 1700 \\
\text { [90] } \\
\text { II. Gibaldone de' soggetti da recitarsi all'improvviso, } \\
\text { alcuni propri e gl'altri da diversi... di Casamarciano } \\
\text { [93] } \\
\text { Napoli, Biblioteca Nazionale, XI.AA.41 e XI.AX.40 } \\
\text { per I. v. Benedetto Croce, Una nuova raccolta di } \\
\text { scenari, in Giornale Storico della Letteratura Italiana, } \\
\text { XXIX, 1 sem. 1897; } \\
\text { per II. v. C. Levi, in “Rivista Teatrale Italiana", XV, } \\
\text { 1911 (sc. 21, 86), XVI, } 1912 \text { (sc. 36, 39), XVIII, 1914 } \\
\text { (sc. a. 30) }\end{array}$ \\
\hline $\begin{array}{l}\text { c. } \\
1700\end{array}$ & [Roma, Vaticana] & 12 & Roma, Biblioteca Vaticana, codex lat., 10244 \\
\hline 1734 & $\begin{array}{l}\text { Perugia, } \\
\text { Comunale] } \\
\text { Placido Adriani } \\
\text { Selva ovvero } \\
\text { zibaldone di } \\
\text { concetti comici... }\end{array}$ & 22 & $\begin{array}{l}\text { Selva ovvero zibaldone di concetti comici... D. Placido } \\
\text { Adriani } \\
\text { Perugia, Biblioteca Comunale, A. } 20 \\
\text { (v. Suzanne Thérault, La commedia dell'arte vue à } \\
\text { travers le zibaldone de Perouse. Etude suivie d'un } \\
\text { choix de scenari de Placido Adriani. Paris: Editions du } \\
\text { C.N.R.S., 1965; e Valentina Gallo, La Selva di Placido } \\
\text { Adriani. La commedia dell'arte nel Settecento. Roma: } \\
\text { Bulzoni, 1998) }\end{array}$ \\
\hline $\begin{array}{l}1722, \\
1743\end{array}$ & $\begin{array}{l}\text { [Parigi, Nationale] } \\
\text { Luigi Riccoboni, } \\
\text { Discorso della } \\
\text { commedia... }\end{array}$ & 6 & $\begin{array}{l}\text { Parigi, Bibliothèque Nationale, Dépt. de la Musique, } \\
\text { Rés. Th.B 102(8-14) } \\
\text { (in L. Riccoboni, Discorso della commedia } \\
\text { all'improvviso e scenari inediti. A cura di I. Mamczarz. } \\
\text { Milano: Il Polifilo, 1973) }\end{array}$ \\
\hline
\end{tabular}




\begin{tabular}{|c|c|c|c|}
\hline \multicolumn{4}{|c|}{ Continuação } \\
\hline XVIII & $\begin{array}{l}\text { Scénario } \\
\text { Biancolelli }\end{array}$ & 80 & $\begin{array}{l}\text { Traduction du scénario de... Biancolelli... par M. G. } \\
\text { [Gueullette] } \\
\text { Parigi, Bibliothèque de l'Opéra, ms. Rés. } 625 \\
\text { (oggi in Delia Gambelli, Arlecchino a Parigi. Roma, } \\
\text { Bulzoni: } 2000,2 \text { v.) }\end{array}$ \\
\hline XVIII & $\begin{array}{l}\text { Copie de la } \\
\text { traduction du } \\
\text { scénario... }\end{array}$ & 72 & $\begin{array}{l}\text { Copie de la traduction du scénario..., Parigi: } \\
\text { Bibliothèque Nationale, f.f. } 9328\end{array}$ \\
\hline $\begin{array}{l}1733, \\
1735\end{array}$ & Scenari "russi" & 39 & $\begin{array}{l}\text { Scenari in russo e tedesco, rappresentati alla corte di } \\
\text { Anna Ioannovna, pubblicati a San Pietroburgo tra il } \\
1733 \text { e il 1735; in Vladimir Nikolaevic Peretts, } \\
\text { Ital'ianskie komedii i intermedii predstavlennye pri } \\
\text { dvore Imperatricy Anny Ioannovny v 1733-35 godack. } \\
\text { Teksty (Commedie e intermezzi italiani rappresentati } \\
\text { alla corte dell'imperatrice Anna Ioannovna negli anni } \\
\text { 1733-1735). Petrograd: Impearatorskaia Akademia } \\
\text { Nauk, 1917 }\end{array}$ \\
\hline \multirow[t]{3}{*}{$\begin{array}{l}1748 \\
1756\end{array}$} & $\begin{array}{l}\text { Scenari per Dresda } \\
\text { e Varsavia }\end{array}$ & 125 & 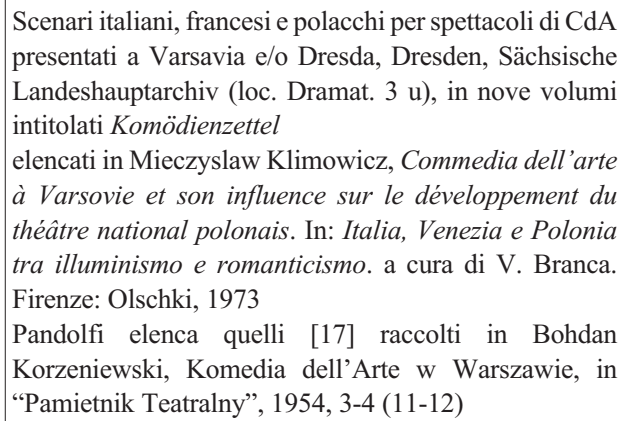 \\
\hline & Scenari isolati & 16 & $\begin{array}{l}\text { Elencati in V. Pandolfi, La commedia dell'arte... cit., } \\
\text { vol. XIII }\end{array}$ \\
\hline & Scenari goldoniani & 29 & In: C. Goldoni, Tutte le opere, a cura di G. Ortolani \\
\hline
\end{tabular}


Per ottobre-novembre, 20 cartelle di 30 righe $x 60$ battute $(20 \times 1800-2000$ caratteri) - ridurre l'argomento

Canovacci. Alle origini della scrittura teatrale moderna. I circa 850 canovacci di commedia dell'arte oggi inventariati costituiscono un corpus di documenti scarsamente studiato nella sua globalità e molto eterogeneo. Appunto in questa eterogeneità sta la sua ricchezza: un ventaglio di exempla di scrittura teatrale che attraversa i secoli, le culture attoriche e soprattutto si articola ogni volta in modo diverso con la scena: in modo riassuntivo, progettuale, allusivo, tra de-scrizione e pre-scrizione, elaborando gerghi espliciti e impliciti, affermando di fatto una scienza della costruzione dello spettacolo che è alla base della scrittura teatrale di tre secoli.

"Soggetti lombardi, lazzi napoletani": contrasto fra narrazione e digressione "Argomento", "soggetto" e "lazzo": la progressiva articolazione del racconto Dalla fisiologia della maschera alla scrittura - Il canovaccio, luogo dove l'esperienza si fa progetto - Esempi di canovacci del seicento, settecento e ottocento: analisi comparata della tecnica di scrittura.

\section{Pandolfi}

Il teatro delle favole rappresentative di Scala è il massimo esempio di "arte spettacolare": "capacità di creare un'ininterrotta vita scenica, un'azione che attragga l'animo dello spettatore nel suo ambito, legandolo alle sue risoluzioni" (v.II, p.74) "siamo come di fronte a una notazione musicale che non può dirci nulla se non viene interpretata" (v.II, p.75). "Naturalmente questo sistema può far pensare ad un arido gioco messo a punto quasi profittando di quelle che sono le perenni reazioni della psiche. Eppure basta un soffio d'umanità conferitogli dall'attore, perché il gioco acquisti una verosimiglianza e un convinzione che gli rendono solidale lo spettatore, divengono strumenti vivificatori della sua fantasia. Il formalismo viene giustificato dagli accenti che lo rendono mezzo, e mezzo eccellentemente temprato" (v.II, p.75).

Secondo V. Pandolfi (La commedia dell'arte... cit., v.II, p.75), fra i molti tipi di scrittura della "commedia dell'arte" (a soggetto, estesa, per la scena, commissionata, consuntiva) gli scenari, di cui alcuni riflessi si trovano in Shakespeare, Molière, Goldoni, non si possono giudicare con criteri letterari, essendo piuttosto paragonabili oggi alla pochade, al circo, agli chansonniers; fin dal principio in essi si prepara l'evoluzione e l'attualizzazione dell'erudita. Quanto alle principali raccolte, ne abbozza una valutazione panoramica: quelle di Scala e Biancolelli sono le due dalla paternità professionale più certa; gli scenari polacchi furono pure di 
professionisti ma meno affermati; i napoletani sembrano avere collegamenti con quelli di Biancolelli, tramite qualche attore; tutti gli altri sembrano o di dilettanti o di piccole compagnie. Locatelli incrementa l'interesse per i moduli romanzeschi, Tirso, Lope, Calderon; Ciro Monarca ha più il gusto della tragedia storica, mentre la commedia vi è quasi scomparsa; la corsiniana riprende temi di entrambe; ma in generale tipica di questo periodo è la scoperta della tragedia. Le altre raccolte (vaticane, magliabechiana, ecc.) non offrono variazioni di interesse Correr svolta verso il larmoyant, prima dei francesi e un secolo prima di Goldoni: precorre il mélo ottocentesco I canovacci della Comédie Italienne: tutta comicità, con vene di patetico Goldoni: il sentimentale e l'allusione.

Hic, nunc et ciak!

Rapporti con la scrittura per il cinema.

$($ scenario $=$ soggetto + sceneggiatura $)$

Paragone del film, dove non è dominante né il gesto né il testo, ma l'Immagine prodotta, nel senso più lato e culturale: non fotografia, ma fotogramma, seppure a passo ridotto; unità elementare di un racconto per Immagini.

\section{L'indipendenza dalla lingua}

Consente lo sperimentalismo lessicale, l'uso anche topico dei diversi linguaggi, la possibilità di farsi comprendere anche da uno spettatore di madre lingua diversa.

CUPPONE, R. "In this", the theater and the scenery of the commedia dell'arte. Trans/Form/Ação (São Paulo), v.24, p.121-141, 2001.

- ABSTRACT: The secret of the Commedia dell'Arte lies in the paradox between Mask and Improvisation, Fixed Character and Variable Text, Conservation and Innovation. Therefore along the centuries it has been exalted or vilified: considered as reactionary during the French revolution and praised by the romantics. In the $20^{\text {th }}$ century Copeau, Meierhold, Mnouchkine and Fo faced this ambivalence. Is that which is today called commedia dell'arte "reactionary" or "revolutionary"? "Popular" or "populist"?

- KEYWORDS: Commedia dell'arte; mask; improvisation. 


\section{Da consultare}

BARTOLI, A. Scenari inediti della CdA. Firenze: Sansoni 1880. (Anast. Forni, 1979)

BARBIERI, N. La supplica. Venezia: Ginammi, 1634. (Ed. critica a cura di F. Taviani, 1971).

BRAGAGLIA, A. G. Commedia dell'Arte: canovacci della gloriosa commedia dell'arte. Raccolti e presentati da Anton Giulio Bargaglia. Torino: Ed. del Dramma S.E.T., 1943. (Cont. 9 scenari della Casanatense).

. La maschera mobile. Foligno: Campitelli 1926.

BRUNI, D. I prologhi. Torino, 1621.

CECCHINI, P. M. Brevi discorsi intorno alle commedie. Napoli: Rocagliolo, 1606. (2.ed. Venezia: Pinelli, 1622).

. Frutti delle moderne commedie. Padova: Guareschi, 1628.

. Pier Maria Cecchini: le commedie... A cura di C. Molinari. Ferrara: Bovolenta, 1983. 211p.

COLAJANNI, G. Les scénarios franco-italiens du ms. 9329 de la Bibliothèque Nationale. Roma: Ed. di Storia e Letteratura, 1970.

PERRUCCI, A. Dell'arte rappresentativa premeditata e all'improvviso. Napoli: Mutio, 1699. (Ed. mod. a cura di A. G. Bragaglia).

PETRACCONE, E. La commedia dell'Arte: storia, tecnica, scenari. Napoli: Ricciardi, 1927.

RE EMILIO. Scenari modenesi. Giornale Storico della Letteratura Italiana, LV, 1910, p. 2 e ss.

RICCOBONI, L. Discorso della commedia all'Improvviso e scenari inediti: a cura di Irene Mamczarz. Milano: Il Polifilo, 1973.

SCALA, F. Il teatro della favole rappresentative: a cura di F. Marotti. Milano: Il Polifilo, 1974.

SPADA, S. Domenico Biancolelli ou l'art d'improviser... Naples: Institut Universitaire Oriental, 1969. 